Journal of Applied Analysis

Vol. 7, No. 2 (2001), pp. 175-190

\title{
EXCEPTIONAL SETS FOR UNIVERSALLY POLYGONALLY APPROXIMABLE FUNCTIONS
}

\author{
M. J. EVANS and P. D. HUMKE \\ Received February 22, 2000 and, in revised form, May 10, 2001
}

\begin{abstract}
In [9], the present authors and Richard O'Malley showed that in order for a function be universally polygonally approximable it is necessary that for each $\varepsilon>0$, the set of points of non-quasicontinuity be $\sigma-(1-\varepsilon)$ symmetrically porous. The question as to whether that condition is sufficient or not was left open. Here we prove that if a set, $E=\bigcup_{n=1}^{\infty} E_{n}$, such that each $E_{i}$ is closed and 1-symmetrically porous, then there is a universally polygonally approximable function, $f$, whose set of points of non-quasicontinuity is precisely $E$. Although it is tempting to call this a partial converse to our earlier theorem it might be more since it is not known if these two notions of symmetric porosity differ in the class of $\mathcal{F}_{\sigma}$ sets.
\end{abstract}

\section{Introduction and definitions}

The purpose of this paper is a straightforward attempt to provide a characterization of an exceptional set of points for a certain class of polygonally approximable functions.

2000 Mathematics Subject Classification. 26A15, 26 A21.

Key words and phrases. Polygonal approximation, polygonally interpolate, interpolate, symmetric porosity, porous set.

ISSN 1425-6908 C Heldermann Verlag. 
We begin by giving a few necessary definitions and some background information. In [1], Agronsky, Ceder, and Pearson showed that any Baire class one function $f:[0,1] \rightarrow \mathbb{R}$ can be represented as the pointwise limit of a sequence of polygonal functions whose vertices lie on the graph of $f$. This result is interesting in several ways, not the least of which is that it shows that, at least in a theoretical sense, linear interpolation of the values of the function can be used to generate a sequence of polygonal functions which converges pointwise to a given, but otherwise arbitrary Baire one function.

In [9], the present authors and Richard O'Malley investigated the subclass of Baire class one functions which have the additional property that for every dense subset $D$ of $[0,1]$, the first coordinates of the vertices of the polygonal functions can be chosen from $D$. As it turns out, this is a real restriction on the Baire one functions and that perhaps, is no surprise, for it is within this class that the aforementioned interpolation can be algorithmically used to generate the sequence of polygonal approximates. It is important to note that in both papers [1] and [9], the sequence of polygonal functions was required to converge pointwise everywhere to the given $f$.

The relevant definitions follow. First we define the subclass, UPA, of Baire one functions we are concerned with in the sequel, next we define the porosity notions relevant to the discussion and finally we state our main result.

a) We say that a function $h:[0,1] \rightarrow \mathbb{R}$ is a polygonal function for $f$ if there is a partition $\tau=\left\{0=a_{0}<a_{1}<a_{2}<\cdots<a_{m}=1\right\}$ such that $h$ agrees with $f$ at each partition point and is linear on the intervening closed intervals. We call $a_{0}, a_{1}, \ldots, a_{m}$ the nodes of $h$ and $\left(a_{0}, h\left(a_{0}\right)\right),\left(a_{1}, h\left(a_{1}\right)\right), \ldots,\left(a_{m}, h\left(a_{m}\right)\right)$ the vertices of $h$. The maximum distance between nodes is called the mesh of $h$ and is denoted $\operatorname{mesh}(h)$.

b) If $S \subset[0,1]$, we say that a sequence $\left\{h_{n}\right\}$ of polygonal functions for $f$ polygonally approximates $f$ on $S$ if $\lim _{n \rightarrow \infty} h_{n}(x)=f(x)$ for every $x \in S$ and $\lim _{n \rightarrow \infty} \operatorname{mesh}\left(h_{n}\right)=0$. In this case we say that $f$ is polygonally approximable on $S$, and if $S=[0,1]$ we say that $f$ is polygonally approximable. Further, if all the nodes of the polygonal functions, other than 0 and 1 , belong to the set of points of continuity, $C(f)$, we say that $\left\{h_{n}\right\} C(f)$-polygonally approximates $f$.

c) $f$ is universally polygonally approximable (UPA) if for every dense subset $D$ in $[0,1]$ there is a sequence $\left\{h_{n}\right\}$ of polygonal functions for $f$, having nodes in $D \cup\{0,1\}$ which polygonally approximates $f$ on $[0,1]$.

The notion of quasicontinuity plays an important role in the understanding of the class UPA and determines the exceptional set which is the focus of the present paper. 
d) A function $f:[0,1] \rightarrow \mathbb{R}$ is quasicontinuous at $x$ if every neighborhood of $(x, f(x))$ contains a point of continuity of $f$. We let $Q(f)$ denote the set of points of quasicontinuity of $f$ and $N Q(f)=(0,1) \backslash Q(f)$.

The exceptional behavior we use here is that of set porosity. If $A$ is a subset of the real line $\mathbb{R}$ and $x \in \mathbb{R}$, then

e) the porosity of $A$ at $x$ is defined to be

$$
\mathrm{p}(A, x)=\limsup _{r \rightarrow 0^{+}} \frac{\lambda(A, x, r)}{r},
$$

where $\lambda(A, x, r)$ is the length of the longest open interval contained in either $(x, x+r) \cap A^{c}$ or $(x-r, x) \cap A^{c}$ and $A^{c}$ denotes the complement of $A$.

f) A set, $A$, is said to be porous at $x$ if $\mathrm{p}(A, x)>0$ and is called a porous set if it is porous at each of its points.

g) The symmetric porosity of $A$ at $x$ is defined as

$$
\operatorname{sp}(A, x)=\limsup _{r \rightarrow 0^{+}} \frac{\gamma(A, x, r)}{r},
$$

where $\gamma(A, x, r)$ is the supremum of all positive numbers $h$ such that there is a positive number $t$ with $t+h \leq r$ such that both of the intervals $(x-t-h, x-t)$ and $(x+t, x+t+h)$ lie in $A^{c}$.

h) A set $A$ is symmetrically porous if $\operatorname{sp}(A, x)>0$ for each $x \in A$. For $0<$ $p \leq 1$ a set $A$ is called $p$-porous ( $p$-symmetrically porous) if $\mathrm{p}(A, x) \geq p$ $(\operatorname{sp}(A, x) \geq p)$ for every $x \in A$.

Symmetrically porous sets are known to be plentiful; indeed, in the space of all compact subsets of $[0,1]$ endowed with the Hausdorff metric, the typical set (in the sense of Baire category) is known to be 1-symmetrically porous (see [16] or [18]). Just as the notion of porosity has proven useful in describing sets of exceptional behavior in real analysis, so too is symmetric porosity beginning to be used to describe exceptionality (e.g., [21], [7], [6]).

Porous sets and symmetrically porous sets have been contrasted in [8], $[17],[11],[12],[13]$ and [20] and, in general, symmetric porosity proves to be a much more restrictive and rigid notion. In [9, Theorem 4] these notions come together in the following way.

Theorem A. Suppose $f:[0,1] \rightarrow \mathbb{R}$ is UPA. Then for each $\varepsilon>0, N Q(f)$ is $\sigma-(1-\varepsilon)$-symmetrically porous, and in fact, can be written as $N Q(f)=$ $\bigcup_{n=1}^{\infty} F_{n}$ where each $F_{n}$ is compact and $(1-\varepsilon)$-symmetrically porous.

The purpose of the present paper is to explore the converse of Theorem A and here we prove: 
Theorem B. Suppose $E=\bigcup_{n=1}^{\infty} F_{n} \subset(0,1)$ where for each $n \in \mathbb{N}, F_{n}$ is compact and 1-symmetrically porous. Then there is a UPA function $f$ : $[0,1] \rightarrow[0,1]$ for which $E=N Q(f)$.

The combination of these two results is interesting for several reasons. First, it is not at all clear from the outset that symmetric porosity has any connection whatsoever with polygonal approximation or the class UPA; yet these theorems show its role to be central. Second, it is known [8], that there is a Borel set (indeed a $G_{\delta}$ ) which is $\sigma-(1-\varepsilon)$-symmetrically porous for every $\varepsilon>0$, but which is not $\sigma-1$-symmetrically porous. But, it is not known if such an $F_{\sigma}$ set exists. So, a solution to this last problem could be exactly what is needed to characterize the points of nonquasicontinuity of UPA functions. In any case, it would be worth some effort to solve this problem.

We continue in the next section with some structural results regarding symmetric perfect sets, and then use those results in the final section where we prove our main result.

\section{A covering theorem}

Suppose $E$ is perfect and 1-symmetrically porous. A component cover of $E$ is a finite set of disjoint closed intervals whose endpoints are in $E$ and whose union contains $E$; unless otherwise specified, the component intervals of such a cover will be indexed in the same order as their relative position on $\mathbb{R}$. For $\varepsilon>0$, an $\varepsilon$-component cover of $E$ is a component cover, $C=\left\{I_{0}, I_{1}, \ldots I_{N}\right\}$, such that

$$
\varepsilon \cdot d\left(I_{n}, I_{n+1}\right)>\max \left(\left|I_{n}\right|,\left|I_{n+1}\right|\right) \text { for } n=1,2, \ldots, N-1
$$

where $d(I, J)$ denotes the distance between the intervals $I$ and $J$. The norm of $C$ is $\delta(C)=\max _{0 \leq n \leq N}\left|I_{n}\right|$. Note that if $\varepsilon<\varepsilon_{0}$ then an $\varepsilon$-component cover is also an $\varepsilon_{0}$-component cover.

In this section we prove a covering property of 1-symmetrically porous sets which is used in the next section to construct a function and polygonal approximations to that function. First, suppose that $E \subset(0,1)$ is 1-symmetrically porous and compact, and let $C$ be a component cover of $E$. If $P$ is any portion of $E$, then

$$
\left.C\right|_{P}=\{[\min (I \cap P), \max (I \cap P)]: I \in C \text { and } I \cap P \neq \emptyset\} .
$$

It is easy to see that in such a situation, $\left.C\right|_{P}$ is a component cover of $P$. We are interested in a sequence of covers, $C_{n}$, of $E$ such that $C_{n+1}$ refines $C_{n}$ for each $n=1,2, \ldots$ in the sense that each component of $C_{n+1}$ is contained in a component of $C_{n}$. 
If $\ell>0$ and $I$ is an interval, $\ell * I$ will denote that interval of length $\ell \cdot|I|$ which is concentric with $I$. If $C$ is a finite set of disjoint closed intervals and $\ell>0$, we define $\ell * C=\bigcup_{I \in C} \ell * I$. The aforementioned covering property can now be described.

Lemma 1. Suppose $\delta>0$ and $0<\varepsilon<1 / 10$ are given and $E$ is a compact $(1-\varepsilon)$-symmetrically porous set. Then there is a $3 \cdot \varepsilon$-component cover $C$ of $E$ with $\delta(C)<\delta$.

Proof. Let $x \in E$. As $E$ is $(1-\varepsilon)$-symmetrically porous there are $t, h>0$ such that $t<\delta / 2,1-1.25 \varepsilon<h /(t+h)$ and $(x-t-h, x-t) \cup(x+t, x+t+h) \subset$ $E^{c}$. Let

$$
I_{x}=[\min (E \cap[x-t, x+t]), \max (E \cap[x-t, x+t])] .
$$

It follows that

$$
\frac{\left|I_{x}\right|}{h}<\frac{2 t}{h}<\frac{2 \varepsilon}{1-\varepsilon}<\frac{20 \varepsilon}{9}<3 \varepsilon
$$

and, of course that $\left|I_{x}\right|<\delta$. As $\varepsilon<1 / 10$, it follows that if $x, y \in E$, either

$$
I_{x} \cap I_{y}=\emptyset \text {, or } I_{x} \subset I_{y} \text { or } I_{y} \subset I_{x} .
$$

The set of these portions, $\left\{I_{x}: x \in E\right\}$ is an open cover for $E$ (in the relative topology) and hence, there is a finite subcover, $\left\{I_{x_{n}}: n=1,2, \ldots, N\right\}$ which, due to (1) we may assume to be mutually exclusive. Reordering this subcover according to the natural order of $\mathbb{R}$ finishes the proof.

Theorem 1. Suppose $E \subset(0,1)$ is perfect and 1-symmetrically porous, and suppose $\left\{\varepsilon_{n}\right\} \rightarrow 0$ is given. Then there exists a sequence of $\varepsilon_{n}$-component covers $\left\{C_{n}\right\}$ of $E$ such that for each $n \in \mathbb{N}$

1. $C_{n+1}$ refines $C_{n}$,

2. $\delta\left(C_{n}\right)<\frac{1}{2^{n+1}}$

3. $2 * C_{1} \subset(0,1),(n+1) * C_{n+1} \subset\left(1+10^{-n}\right) * C_{n}$.

Proof. The proof consists of applying Lemma 1 inductively. We assume that $\varepsilon_{n}<1 / 10$ in all cases.

1. According to Lemma 1 , since $E$ is $\left(1-\varepsilon_{1} / 3\right)$-symmetrically porous, there is an $\varepsilon_{1}$-component cover $C_{1}$ of $E$ with $\delta\left(C_{1}\right)<1 / 4$.

2. Now assume an $\varepsilon_{n}$-component cover $C_{n}$ of $E$ is given with $\delta\left(C_{n}\right)<$ $1 /\left(2^{n+1}\right)$ and let $I$ be a fixed element (interval) from $C_{n}$. As $I \cap$ $E$ is 1 -symmetrically porous we can apply Lemma 1 to obtain an $\varepsilon_{n+1}$ - component cover $C(I)$ of $I \cap E$ such that

$$
\delta(C(I))<\frac{|I|}{(n+1) \cdot 10^{n}} .
$$


This condition entails two separate aspects of the relationship between $I$ and $C(I)$ which are technically important to our subsequent application of Theorem 1. These are

$$
\begin{gathered}
(n+1) * C(I) \subset\left(1+\frac{1}{10^{n}}\right) * I \\
\delta(C(I))<\frac{1}{2^{n+2}} .
\end{gathered}
$$

To complete the inductive step we let

$$
C_{n+1}=\bigcup_{I \in C_{n}} C(I) \text {. }
$$

If $I_{1}$ and $I_{2}$ are distinct intervals in $C_{n}$, then it follows from Lemma 1 that the distance between them is longer than either of their lengths. As $E \cap C\left(I_{1}\right)$ consists of portions of $E \cap I_{1}$ and $C\left(I_{1}\right) \subset\left(1+1 / 10^{n}\right) * I_{1}$, it follows that each interval in $C\left(I_{1}\right)$ is contained in $I_{1}$. From this it is easy to see that $C_{n+1}$ refines $C_{n}$. The remaining conditions of the theorem, Conditions \#2 and \#3, follow immediately from this, inclusion (3), and inequality (4). This, then, completes the proof of Theorem 1.

\section{The perfect 1 -symmetrically porous case}

In this section we inductively use Theorem 1 to prove the following, rather delicate Theorem 2. Theorem 2 is the main engine for the final construction detailed in the final section where it is used to construct a series of functions. It is easy to see that this series converges uniformly, but not so easy to see that the limit function is UPA. It is exactly at this juncture that the seemingly extraneous Condition 5 of Theorem 2 plays a critical role.

Theorem 2. Suppose $E \subset(A, B) \subset[A, B] \subset(0,1)$ is 1-symmetrically porous and perfect. Suppose too that $S=\left\{a_{n}, b_{n}\right\} \subset(A, B)$ is a given sequence with $\left[a_{n}, b_{n}\right] \cap E=\emptyset$ for each $n \in \mathbb{N}$. Then, there is a sequence of polygonal functions, $h_{n}:[0,1] \rightarrow[0,1]$ such that

1. $\left\{h_{n}\right\}$ converges pointwise to a function $f:[0,1] \rightarrow[0,1]$,

2. $f(x)=1 / 2$ if $x \in E$,

3. $f$ is non-quasicontinuous at each $x \in E$,

4. $f$ is continuous at each $x \in[0,1] \backslash E$,

5. nodes of $h_{n}$ are in $[0,1] \backslash\left(E \cup \bigcup_{i=1}^{n}\left(a_{i}, b_{i}\right)\right)$ and $\left.f\right|_{\left[a_{i}, b_{i}\right]} \equiv 0($ or $\equiv 1)=$ $\left.h_{n}\right|_{\left[a_{i}, b_{i}\right]}$ whenever $i \leq n$,

6. $f(0)=f(1)=0$. 
Proof. Set $\varepsilon_{n}=10^{-n}$. Using Theorem 1 we first construct a sequence of $\varepsilon_{n}$-component covers of $E, C_{n}$, such that $(n+1) * C_{n} \subset(A, B)$ and $(n+1) * C_{n} \cap\left[a_{n}, b_{n}\right]=\emptyset$ for each $n \in \mathbb{N}$. We define a pointwise convergent sequence of polygonal functions, $\left\{h_{n}\right\}$, using these covers and then define $f$ as the pointwise limit of the $\left\{h_{n}\right\}$. The nodes of $h_{n}$ will either map to 0 or 1 with those mapping to 0 denoted by $N_{n}(0)$ and those mapping to 1 denoted by $N_{n}(1)$.

$h_{1}$ : First, put $0,1,(B+1) / 2 \in N_{1}(0)$; that is, the points 0,1 , and $(B+1) / 2$ will be nodes of $h_{1}$ and will all map to 0 . Suppose $C_{1}=$ $\left\{I_{0}, I_{1}, \ldots I_{N}\right\}$.

Since $C_{1}$ is a $1 / 10$-component cover of $E, d\left(I_{i}, I_{i+1}\right)>10 \cdot \max \left(\left|I_{i}\right|\right.$, $\left.\left|I_{i+1}\right|\right)$ for each $i=0,1, \ldots, N-1$. At each left endpoint of $2 * I_{i}$, define $h_{1}$ to be 0 if $i$ is even, and 1 if $i$ is odd; at right endpoints of $2 * I_{i}, h_{1}$ is just the reverse, 1 if $i$ is even and 0 if $i$ is odd.

Note that $h_{1}$ is constant (either 0 or 1 ) at neighboring nodes bordering a large central portion of the interval between two consecutive $I_{i}$ 's and is not constant between neighboring nodes surrounding an interval $I \in C_{1}$.

If $I \in C_{1}$, then $I$ is called a $(0,1)$-interval if $h_{1}$ is increasing between its neighboring nodes, otherwise $I$ is termed a $(1,0)$-interval. Also, every pair of neighboring nodes on which $h_{1}$ is not constant contains a covering interval, $I_{i} \in C_{1}$, with the possible single exception of the interval extending between $2 * I_{N}$ and $(B+1) / 2$. If $N$ is even, then $h_{1}$ maps the right endpoint of $2 * I_{N}$ to 1 and yet $h_{1}((B+1) / 2)=0$.

At this juncture, the nodes of $h_{1}$ partition $[0,1]$ into three possible types of intervals:

1. intervals on which $h_{1}$ is constant (either 0 or 1 ),

2. (0,1)-intervals or $(1,0)$-intervals which intersect $E$,

3 . possibly a $(1,0)$ interval which does not intersect $E$.

This interval, extant if $N$ is even, extends between the right endpoint of $2 * I_{N}$ and $(B+1) / 2$.

The cover $C_{1}$ was chosen so that it not only misses $\left[a_{1}, b_{1}\right]$, but so that $2 * C_{1}$ misses $\left[a_{1}, b_{1}\right]$. Hence, $\left[a_{1}, b_{1}\right]$ intersects no partition interval of type 2 above. We complete the definition of $h_{1}$ by considering $\left[a_{1}, b_{1}\right]$.

The simplest case is when $N$ is odd and $\left[a_{1}, b_{1}\right]$ lies between consecutive nodes in $N_{1}(0)$ (or consecutive nodes in $N_{1}(1)$ ). In this case, the definition of $h_{1}$ is completed by defining $h_{1}$ to be linear between nodes.

If $N$ is even more must be done. If $N$ is even and $\left[a_{1}, b_{1}\right]$ lies between consecutive nodes in $N_{1}(0)$ (or consecutive nodes in $N_{1}(1)$ ), we define 
$h_{1}$ to to be linear between nodes and designate the interval between $2 * I_{N}$ and $(B+1) / 2$ as a $(1,0)-$ spline interval of Stage 1 .

The remaining case is when $N$ is even and $\left[a_{1}, b_{1}\right]$ lies in the interval between $2 * I_{N}$ and $(B+1) / 2$. In this case, we place both $a_{1}$ and $b_{1}$ in $N_{1}(1)$ and designate the interval $\left[b_{1},(B+1) / 2\right]$ as a $(1,0)-$ spline interval of Stage 1. Again, $h_{1}$ is extended linearly between nodes.

Inductively, suppose that the polygonal function $h_{n}$ has been defined such that the nodes of $h_{n}$ partition $[0,1]$ into intervals of the following types:

1. intervals on which $h_{n}$ is constant.

2. (0,1)-intervals or $(1,0)$-intervals which intersect $E$.

An interval of this type is of the form $(n+1) * J$ where $J$ is one of the covering intervals from $C_{n}$. Moreover, $E$ is contained in the union of the intervals of this category.

3 . (0,1)-spline intervals or $(1,0)$-spline intervals.

If $I$ is a spline interval, then the interval to the right of $I$ is an interval of constancy and the interval to the left of $I$ intersects $E$.

$h_{n+1}$ : The nodes of $h_{n}$ partition $[0,1]$ into three possible types of intervals. We begin by defining $h_{n+1}$ separately on partition intervals of these categories, and then show how to alter this definition to account for $\left[a_{n+1}, b_{n+1}\right]$.

1. If $h_{n}$ is constant on an interval, then we define $h_{n+1}$ to be that same constant.

2. Suppose $I=[a, b]$ is a $(0,1)$-interval which intersects $E$. In this case we proceed in a manner completely analogous to that of $h_{1}$, but entirely within $I$. We begin by establishing the value of $h_{n+1}$ at the endpoints, $a$ and $b$.

As $I$ is a $(0,1)$-interval, $a \in N_{n}(0)$ and we put $a \in N_{n+1}(0)$. We also know that $b \in N_{n}(1)$ and $b$ will be a node of the $n+1^{\text {st }}$ stage, but perhaps not a 1-node. As $h_{n}$ is constant in a neighborhood of 1 , there is a partition interval, $[b, c]$, immediately to the right of $[a, b]$. If that partition interval is of the first type, then $\left.h_{n}\right|_{[b, c]} \equiv 1$ and we put $h_{n+1}(b)=1$. If, however, $h_{n}$ is not of the first type on $[b, c]$, then $[b, c]$ is a spline interval of Stage n. In this case, $\left.h_{n}\right|_{[c, d]} \equiv 0$ where $[c, d]$ is the partition interval immediately right of $[b, c]$ and we define $h_{n+1}(b)=0$.

Consider the cover $C_{n+1}$ restricted to $I$,

$$
\left.C_{n+1}\right|_{I}=\left\{J_{0}, J_{1}, \ldots, J_{m_{n+1}}\right\} .
$$

As $I$ is a $(0,1)$-interval, we declare each left endpoint of $(n+1) * J_{i}$ to be in $N_{n+1}(0)$ if $i$ is even and to be in $N_{n+1}(1)$ if $i$ is odd; at 
the right endpoints this declaration is reversed. (If $I$ is a $(1,0)$ interval, these declarations are interchanged.)

The function $\left.h_{n+1}\right|_{[a, b]}$ is now simply defined to be 0 at points of $N_{n+1}(0) \cap[a, b], 1$ at points of $N_{n+1}(1) \cap[a, b]$, and linear between consecutive points of $N_{n+1}(0) \cup N_{n+1}(1)$.

The nodes of $\left.h_{n+1}\right|_{[a, b]}$ delineate intervals on which $h_{n+1}$ is constant (either 0 or 1 ), intervals on which $h_{n+1}$ is linear and increases from 0 to 1 (these are $(0,1)$-intervals of Stage $n+1)$ and intervals on which $h_{n+1}$ is linear and decreases from 1 to 0 (these are $(1,0)$-intervals of Stage $n+1)$. If $d$ denotes the last node less than $b$, then the interval $[d, b]$ falls into one of these categories and in no case does $[d, b]$ intersect $E$.

3. Suppose $I=[a, b]$ is a spline interval of $h_{n}$. In this case, the partition interval to the right of $I$ is an interval of constancy of $h_{n}$ (either 0 or 1 ) and we define $\left.h_{n+1}\right|_{[a, b]}$ to be that constant. Note that this definition dovetails with the definition of $h_{n+1}$ described in 2 above in the sense that the unrestricted function $h_{n+1}$ is continuous on all of $[0,1]$.

At this point the piecewise linear function, $h_{n+1}$ has been defined on all of $[0,1]$ and because the definition in part 3 above dovetails with that given in parts 1 and $2, h_{n+1}$ is continuous. Moreover, the nodes of $h_{n+1}$ partition [0,1] into three possible types of intervals:

1. intervals on which $h_{n+1}$ is constant (either 0 or 1 ),

2. (0,1)-intervals or $(1,0)$-intervals which intersect $E$,

3 . possible $(0,1)$ or $(1,0)$-intervals which miss $E$.

Moreover, each $(1,0)$-interval which misses $E$ is preceded by a $(0,1)$ interval which intersects $E$ and is followed by an interval on which $h_{n+1}$ is identically 1 . Also, each $(0,1)$-interval which misses $E$ is preceded by a $(1,0)$-interval which intersects $E$ and is followed by an interval on which $h_{n+1}$ is identically 0 .

To complete Stage $n+1$ we alter this definition to account for $\left[a_{n+1}, b_{n+1}\right]$. The cover $C_{n+1}$ was chosen so that $(n+1) * C_{n+1}$ misses $\left[a_{n+1}, b_{n+1}\right]$ and hence, $\left[a_{n+1}, b_{n+1}\right]$ intersects no partition interval of type 2 above.

Case 1. $\left[a_{n+1}, b_{n+1}\right]$ intersects an interval $[c, d]$ of type 3 .

Since $[c, d]$ is of type $3, h_{n+1}$ is constant on a partition interval $[d, e]$ immediately to the right of $[c, d]$. Moreover, since $\left[a_{n+1}, b_{n+1}\right]$ intersects no interval of type $2, c<a_{n+1} \leq d$ and $b_{n+1}<e$. We redefine $h_{n+1}$ on $(c, d)$ as follows:

$$
h_{n+1}(x)= \begin{cases}h_{n+1}(d) & \text { if } a_{n+1} \leq x<d \\ \frac{h_{n+1}(d)-h_{n+1}(c)}{a_{n+1}-c}(x-c)+h_{n+1}(c) & \text { if } c<x<a_{n+1} .\end{cases}
$$


The interval $\left[c, a_{n+1}\right]$ is now deemed a spline interval of Stage $n+1$, and $a_{n+1}$ and $b_{n+1}$ are designated nodes of $h_{n+1}$. If $d<b_{n+1}$, then $d$ is decommissioned as a node of $h_{n+1}$. In any case, $h_{n+1}$ is constant on $\left[a_{n+1}, b_{n+1}\right]$, both $a_{n+1}$ and $b_{n+1}$ are nodes of $h_{n+1}$ and $\left(a_{n+1}, b_{n+1}\right)$ contains no nodes of $h_{n+1}$.

Case 2. $\left[a_{n+1}, b_{n+1}\right]$ does not intersect an interval of type 3 .

In this case, $\left[a_{n+1}, b_{n+1}\right]$ lies entirely within an interval of constancy of $h_{n+1}$ and we alter the definition of $h_{n+1}$ only by designating $a_{n+1}$ and $b_{n+1}$ to be nodes of $h_{n+1}$.

This completes the definition of $h_{n+1}$ and by induction, the sequence, $\left\{h_{n}\right\}$. We proceed to show it has the properties we claimed it did.

First, we show that the sequence, $\left\{h_{n}\right\}$, converges pointwise to a function, $f \in$ UPA. To this end, fix $x_{0} \in[0,1]$. We consider two cases.

Case 1. Suppose $x_{0} \in E$. Then for each $n \in \mathbb{N}$, there is a unique interval $I=[a, b] \in C_{n}$ which contains $x_{0}$. Then $n * I \equiv[\alpha, \beta]=$ $[(a+b) / 2-(n / 2)(b-a),(a+b) / 2+(n / 2)(b-a)]$ and the construction entails that $\left.h_{n}\right|_{[\alpha, \beta]}$ is linear with $h_{n}(\alpha)=0$ and $h_{n}(\beta)=1$ or $h_{n}(\alpha)=1$ and $h_{n}(\beta)=0$. In either case, $h_{n}(I)=[1 / 2-$ $1 /(2 n), 1 / 2+1 /(2 n)]$. It follows that $\left\{h_{n}\left(x_{0}\right)\right\} \rightarrow 1 / 2$.

Case 2. Suppose $x_{0} \notin E$. Then, there is an $N \in \mathbb{N}$ such that $C_{N}$ fails to cover $x_{0}$. As such, $x_{0}$ is either in an interval of constancy of $h_{N}$ or in a spline interval of $h_{N}$. In the first instance, $h_{N}\left(x_{0}\right)=h_{i}\left(x_{0}\right)$ for all $i \geq N$ and hence, $\left\{h_{N}\left(x_{0}\right)\right\}$ converges and converges to either 0 or 1 . In the second instance, the spline interval of $h_{N}$ which contains $x_{0}$ becomes an interval of constancy of $h_{N+1}$ so that $h_{i}\left(x_{0}\right)=h_{N+1}\left(x_{0}\right)$ whenever $i \geq N+1$ and again, $\left\{h_{N}\left(x_{0}\right)\right\}$ converges and again, converges to either 0 or 1 .

We let $f(x)=\lim _{n \rightarrow \infty} h_{n}(x)$. If $x \notin E$, then there is an interval, $I$, contiguous to $E$ which contains $x$. The argument given above in Case 2 shows that either $f(I)=0$ or $f(I)=1$ and in either case, $f$ is continuous at $x$. If $x \in E$, then $f(x)=1 / 2$ so that the ball about $(x, f(x))$ of radius $1 / 4$ contains no point of the graph of $\left.f\right|_{E^{c}}$. From this it is easy to see that $E=N Q(f)$ and $E^{c}=C(f)$.

Conclusion 5 follows from the fact that $h_{n}$ is constant (either 0 or 1 ) on $\left[a_{n}, b_{n}\right]$ and that for $k>n, h_{k}$ is constant on any interval where $h_{n}$ is constant and contains no nodes interior to any interval of constancy.

This completes the proof of Theorem 2; in the next section we use this theorem in an inductive way to construct a function whose points of non-quasicontinuity is a given countable union of compact 1-symmetrically porous sets. The construction is complicated by the fact that UPA is not closed under uniform limits. 


\section{The $\sigma$ case}

We begin with four lemmas. The first two of these are transparent but are recorded for completeness. The third lemma is technical in nature and sets out conditions under which a certain uniform sum of UPA functions is UPA. The final lemma is a standard disengagement result for unions of compact 1-symmetrically porous sets.

For each polygonal function $h$, set $N(h)=\{x \in[0,1]: h$ has a node at $x\}$.

Lemma 2. If $E=\left\{x_{0}\right\} \subset(0,1)$ and $S=\left\{a_{n}, b_{n}\right\}$ is a given sequence such that $x_{0} \notin\left[a_{n}, b_{n}\right]$ for every $n \in \mathbb{N}$, then there is a sequence of polygonal functions, $h_{n}:[0,1] \rightarrow[0,1]$ such that

1. $\left\{h_{n}\right\}$ converges pointwise to a function $f:[0,1] \rightarrow[0,1]$,

2. $f\left(x_{0}\right)=1 / 2$,

3. $f$ is non-quasicontinuous at $x_{0}$,

4. $f$ is continuous at each $x \in[0,1] \backslash E$,

5. nodes of $h_{n}$ are in $[0,1] \backslash \bigcup_{i=1}^{n}\left[a_{i}, b_{i}\right]$ and $\left.f\right|_{\left[a_{i}, b_{i}\right]} \equiv 0($ or $\equiv 1)=$ $\left.h_{n}\right|_{\left[a_{i}, b_{i}\right]}$ whenever $i \leq n$,

6. $f(0)=f(1)=0$.

Proof. Let $4 \delta_{n}=\min \left(\left|x_{0}-a_{i}\right|,\left|x_{0}-b_{i}\right|\right), i \leq n$, and define

$$
h_{n}(x)= \begin{cases}0 & x \notin\left(x_{0}-\delta_{n}, x_{0}+3 \delta_{n}\right) \\ 1 & x=x_{0}+\delta_{n} \\ \text { linearly on } & {\left[x_{0}-\delta_{n}, x_{0}+\delta_{n}\right] \text { and }\left[x_{0}+\delta_{n}, x_{0}+3 \delta_{n}\right] .}\end{cases}
$$

Lemma 3. Suppose that for each fixed $n \in \mathbb{N}$ we have:

1. $\left\{h_{n, m}\right\}$ converges pointwise to $f_{n}$,

2. $h_{n, m}$ is polygonal with vertices on $\left.f_{n}\right|_{C\left(f_{n}\right)}$,

3. if $1 \leq n \leq m$ and $h_{n, m}$ has a node at $x^{*}$, then $h_{k, m}\left(x^{*}\right)=f_{k}\left(x^{*}\right)$ whenever $1 \leq k \leq m$,

4. $\left|h_{n, m}\right| \leq 1 / 2^{n}$ for all $m \in \mathbb{N}$.

Then $\sum f_{n} \in \mathrm{UPA}$.

Proof. Fix $n$ and define

$$
g_{n}(x)=h_{1, n}(x)+h_{2, n}(x)+\cdots+h_{n, n}(x) \text { and } f(x)=\Sigma f_{n}(x) .
$$

A. $\left\{g_{n}\right\} \rightarrow f$ pointwise. 
Fix $x_{0} \in[0,1]$ and let $\varepsilon>0$ be given. Then there is an $N \in \mathbb{N}$ such that $2^{1-N}<\varepsilon / 2$ and an $M \geq N$ such that for $n=1,2, \ldots, N$ and $m \geq M$,

$$
\left|h_{n, m}(x)-f_{n}(x)\right|<\frac{\varepsilon}{2 N} .
$$

Now, for $m \geq M$

$$
\begin{aligned}
\left|g_{m}(x)-f(x)\right| & \leq \sum_{i=1}^{N}\left|h_{i, m}(x)-f_{i}(x)\right|+\sum_{i=N+1}^{\infty}\left(\left|h_{i, m}(x)\right|+\left|f_{i}(x)\right|\right) \\
& \leq N \frac{\varepsilon}{2 N}+2 \frac{1}{2^{N}}<\varepsilon .
\end{aligned}
$$

B. If $x^{*} \in N\left(g_{n}\right)$, then $\left|g_{n}\left(x^{*}\right)-f\left(x^{*}\right)\right| \leq 1 / 2^{n}$.

Let $x^{*} \in N\left(g_{n}\right) \equiv \bigcup_{m=1}^{n} N\left(h_{m, n}\right)$. Then there is an $1 \leq m \leq n$ with $x^{*} \in N\left(h_{m, n}\right)$ and it follows from hypothesis 3 that $h_{k, n}\left(x^{*}\right)=f_{k}\left(x^{*}\right)$ for all $1 \leq k \leq n$. It now follows that

$$
\begin{aligned}
\left|g_{n}\left(x^{*}\right)-f\left(x^{*}\right)\right| & =\left|\sum_{k=1}^{n} h_{k, n}\left(x^{*}\right)-\sum_{k=1}^{\infty} f_{k}\left(x^{*}\right)\right| \\
& =\left|\sum_{k=1}^{n} f_{k}\left(x^{*}\right)-\sum_{k=1}^{\infty} f_{k}\left(x^{*}\right)\right| \\
& \leq \sum_{k=n+1}^{\infty}\left|f_{k}\left(x^{*}\right)\right| \leq \frac{1}{2^{n}} .
\end{aligned}
$$

Define $g_{n}^{*}(x)=\left\{\begin{array}{l}f(x) \text { if } x \in N\left(g_{n}\right) \\ \text { linear between consecutive nodes. }\end{array}\right.$

C. $\left\{g_{n}^{*}\right\} \rightarrow f$ pointwise on $[0,1]$.

From the definition of $g^{*}$ and $\mathbf{B}$ it follows that $\left|g_{n}^{*}(x)-g_{n}(x)\right|=\mid f(x)-$ $g_{n}(x) \mid \leq 1 / 2^{n}$ whenever $x \in N\left(g_{n}\right)$ and consequently that $\left|g_{n}^{*}(x)-g_{n}(x)\right| \leq$ $1 / 2^{n}$ uniformly on $[0,1]$. From this and the fact that $\left\{g_{n}\right\} \rightarrow f$ pointwise, it follows that $\left\{g_{n}^{*}\right\} \rightarrow f$ pointwise. But, by [9, Proposition 1], $f$ is UPA if and only if it is $C(f)$-polygonally approximable so this completes the proof of the lemma.

Lemma 4. Suppose $E=E_{1} \cup E_{2} \cup \ldots$ where each $E_{n}$ is either perfect and 1-symmetrically porous or a singleton. Then $E=E_{1}^{*} \cup E_{2}^{*} \cup \ldots$ where each $E_{n}^{*}$ is perfect and 1-symmetrically porous or a singleton, and for each $n \in \mathbb{N}$, $E_{n+1}^{*}$ lies in a single component of $[0,1] \backslash \bigcup_{i=1}^{n} E_{i}^{*}$. 
Proof. Let $E_{0}=\emptyset$. For each $n \in \mathbb{N}, E_{n} \backslash E_{n-1}$ is 1-symmetrically porous and is an $F_{\sigma}$ set. As such, $E_{n} \backslash E_{n-1}$ can be written as the countable, disjoint union of compact, 1-symmetrically porous sets. Hence, there are disjoint, compact, 1-symmetrically porous sets, $F_{n}$, such that $\bigcup_{n=1}^{\infty} E_{n}=\bigcup_{n=1}^{\infty} F_{n}$.

Define $E_{1}^{*}=F_{1}$ and let $k \in \mathbb{N}$. As $F_{k+1} \cap \bigcup_{i=1}^{k} F_{i}=\emptyset$, and $F_{k+1}$ is compact, only finitely many components of $[0,1] \backslash \bigcup_{i=1}^{k} F_{i}$ intersect $F_{k+1}$ and we enumerate those portions of $F_{k+1}$ as $E_{n_{k}+1}^{*}, E_{n_{k}+2}^{*}, \ldots, E_{n_{k+1}}^{*}$. The conclusion now follows.

Theorem 3. Suppose $E=\bigcup_{n=1}^{\infty} F_{n} \subset(0,1)$ where for each $n \in \mathbb{N}, F_{n}$ is compact and 1-symmetrically porous. Then there is a UPA function $f$ : $[0,1] \rightarrow[0,1]$ for which $E=N Q(f)$.

Proof. Apply Lemma 4 to write $E=E_{1}^{*} \cup E_{2}^{*} \cup \ldots$ where each $E_{n}^{*}$ is perfect and 1-symmetrically porous or a singleton, and for each $n \in \mathbb{N}, E_{n+1}^{*}$ lies in a single component of $[0,1] \backslash \bigcup_{i=1}^{n} E_{i}^{*}$. In the remainder of the proof, we use the basic constructions of Lemma 2 and Theorem 2 in an inductive manner to obtain sequences of polygonal functions, $\left\{h_{n, m}\right\}$, satisfying the conditions of Lemma 3. If $A \subset[0,1]$, we denote the closed convex hull of $A$ by hull $(A)$.

For each $n \geq 2, \operatorname{hull}\left(E_{n}^{*}\right) \subset[0,1] \backslash \bigcup_{i=1}^{n-1} E_{i}^{*}$, and so there are $a_{n-1}<b_{n-1}$ such that $E_{n}^{*} \subset\left(a_{n-1}, b_{n-1}\right) \subset\left[a_{n-1}, b_{n-1}\right] \subset[0,1] \backslash \bigcup_{i=1}^{n-1} E_{i}^{*}$ and neither $a_{n-1}$ nor $b_{n-1} \in E$. We proceed inductively.

STEP 1. We use the basic constructions to define a sequence $h_{1, m}$ with $E=E_{1}^{*}$ and $S=\left\{a_{n}, b_{n}: n=1,2, \ldots\right\}$. Then, $\left\{h_{1, m}\right\}$ converges pointwise on $[0,1]$ to a function $f_{1}$ with $f_{1}(x)=1 / 2$ whenever $x \in$ $E_{1}^{*}$ and $f_{1}$ is constant (either 0 or 1 ) on each interval contiguous to $E_{1}^{*}$. Moreover, if $i=1,2, \ldots, m$, then $\left.h_{1, m}\right|_{\left[a_{i}, b_{i}\right]}$ is constant (again, either 0 or 1 ) and that constant coincides with $f_{1}\left(\left[a_{i}, b_{i}\right]\right)$. This completes the construction of the sequence, $\left\{h_{1, m}\right\}$, and its pointwise limit, $f_{1}$.

STEP $n$. Suppose now that $n \geq 2$. We use the basic constructions to define a sequence $h_{n, m}$ with $E=E_{n}^{*}$ and $S=\left\{a_{k}, b_{k}: k=n, n+1, \ldots\right\}$, in such a way that $h_{n, m} \equiv 0$ outside of the containment interval, $\left[a_{n-1}, b_{n-1}\right]$.

To this end, let $\ell:\left[a_{n-1}, b_{n-1}\right] \rightarrow[0,1]$ be increasing and linear, mapping $\left[a_{n-1}, b_{n-1}\right]$ onto $[0,1]$ and set $\tilde{E}_{n}=\ell\left(E_{n}^{*}\right)$ and $\tilde{S}=\ell(S)$. Once more, we use the basic constructions to define a sequence, $\left\{\tilde{h}_{n, m}\right\}$, which converges pointwise on $[0,1]$ to a function, $\tilde{f}_{n}$ with $\tilde{f}_{n}(x)=1 / 2$ for $x \in \tilde{E}_{n}$ and $\tilde{f}_{n}$ is constant (either 0 or 1 ) on each interval contiguous to $\tilde{E}_{n}$. Moreover, if $i=n, n+1, \ldots, m$, then 
$\left.\tilde{h}_{n, m}\right|_{\left[a_{i}, b_{i}\right]}$ is constant and that constant coincides with $\tilde{f}_{n}\left(\left[a_{i}, b_{i}\right]\right)$. For $m \geq n$, define

$$
h_{n, m}(x)= \begin{cases}\frac{1}{2^{n}} \tilde{h}_{n, m} \circ \ell(x) & \text { if } a_{n-1} \leq x \leq b_{n-1} \\ 0 & \text { otherwise }\end{cases}
$$

and let $f_{n}$ denote the pointwise limit of $\left\{h_{n, m}\right\}$.

It is easy to see that $\left\{h_{n, m}\right\}$ and $f_{n}$ satisfy conditions 1,2 and 4 of Lemma 3.

To see that they also satisfy 3 , let $x_{0}$ be a node of $h_{n_{0}, m_{0}}$ where $1 \leq$ $n_{0} \leq m_{0}$. We must verify that for $1 \leq i \leq m_{0}$, that $h_{i, m_{0}}\left(x_{0}\right)=$ $f_{i}\left(x_{0}\right)$.

Case $1.1 \leq i<n_{0}$

As $x_{0}$ is a node of $h_{n_{0}, m_{0}}, x_{0} \in\left[a_{n_{0}-1}, b_{n_{0}-1}\right]$ and as $i<n_{0}$, either

$$
\left[a_{n_{0}-1}, b_{n_{0}-1}\right] \subset\left[a_{i-1}, b_{i-1}\right]
$$

or

$$
\left[a_{i-1}, b_{i-1}\right] \subset[0,1] \backslash\left[a_{n_{0}-1}, b_{n_{0}-1}\right] .
$$

In either case, $\left.h_{i, m_{0}}\right|_{\left[a_{n_{0}-1}, b_{n_{0}-1}\right]} \equiv 0$ (or $\left.\equiv 1\right)\left.\equiv f_{i}\right|_{\left[a_{n_{0}-1}, b_{n_{0}-1}\right]}$ since $n_{0}-1<m_{0}$. Since $x_{0} \in\left[a_{n_{0}-1}, b_{n_{0}-1}\right]$ it follows from the basic constructions that $h_{i, m_{0}}\left(x_{0}\right)=f_{i}\left(x_{0}\right)$.

Case 2. $i=n_{0}$

In this case $f_{n_{0}}\left(x_{0}\right)=h_{n_{0}, m_{0}}\left(x_{0}\right)$ since every vertex of $h_{n_{0}, m_{0}}$ lies on the graph of $f_{n_{0}}$.

Case 3. $n_{0}+1 \leq i \leq m_{0}$

The nodes of $h_{n_{0}, m_{0}}$ lie outside $\bigcup_{j=n_{0}}^{m_{0}}\left[a_{j}, b_{j}\right]$, so in particular, $x_{0} \notin\left[a_{i-1}, b_{i-1}\right]$. This implies that $h_{i, m_{0}}\left(x_{0}\right)=0=f_{i}\left(x_{0}\right)$ since $x_{0}$ lies outside the support of $h_{i, m_{0}}$.

This completes the proof of Theorem 3 .

We conclude by restating the question we considered earlier in the Introduction.

Question 1. Suppose that for every $\varepsilon>0, E=\bigcup_{n=1}^{\infty} F_{n}$ where each $F_{n}$ is compact and $(1-\varepsilon)$-symmetrically porous. Is $E \sigma-1$-symmetrically porous?

If the answer to Question 1 is positive, then Theorem 3 provides a complete converse to Theorem A; if however, the answer is negative, more must be done to characterize the set $N Q(f)$. 


\section{References}

[1] Agronsky, S. J., Ceder, J. G., Pearson, T. L., Some characterizations of Darboux Baire 1 functions, Real Anal. Exchange 23 (1997-98), 421-429.

[2] Bruckner, A. M., Differentiation of Real Functions, CRM Monograph Series, Vol. 5, 2nd ed., American Mathematical Society, Providence, 1994.

[3] Ceder, J. G. and Pearson, T. L., A survey of Darboux Baire 1 functions, Real Anal. Exchange 9 (1983-84), 179-193.

[4] Darji, U. B., Evans, M. J., Freiling, C., and O'Malley, R. J., Fine properties of Baire one functions, Fund. Math. 155 (1998), 177-188.

[5] Denjoy, A., Leçons sur le Calcul des Coefficients d'une Série Trigonométrique, Part II, Métrique et Topologie d'Ensembles Parfaits et de Fonctions, Gauthier-Villars, Paris, 1941.

[6] Evans, M. J., Some theorems whose $\sigma$-porous exceptional sets are not $\sigma$-symmetrically porous, Real Anal. Exchange 17 (1991-92), 809-814.

[7] Evans, M. J., A note on symmetric and ordinary differentiation, Real Anal. Exchange 17 (1991-92), 820-826.

[8] Evans, M. J. and Humke, P. D., Contrasting symmetric porosity and porosity, J. Appl. Anal. 4(1) (1998), 19-41.

[9] Evans, M. J., Humke, P. D. and O'Malley, R. J., Universally polygonally approximable functions, J. Appl. Anal. 6(1) (2000), 1-16.

[10] Evans, M. J., Humke, P. D. and O'Malley, R. J., A perplexing collection of Baire one functions, Real Anal. Exchange, (to appear).

[11] Evans, M. J., Humke, P. D. and Saxe, K., A symmetric porosity conjecture of Zajiček, Real Anal. Exchange 17 (1991-92), 258-271.

[12] Evans, M. J., Humke, P. D. and Saxe, K., Symmetric porosity of symmetric Cantor sets, Czechoslovak Math. J. 44 (1994), 251-264.

[13] Evans, M. J., Humke, P. D. and Saxe, K., A characterization of $\sigma$-symmetrically porous symmetric Cantor sets, Proc. Amer. Math. Soc. 122 (1994), 805-810.

[14] Evans, M. J. and O'Malley, R. J., Fine tuning the recoverability of Baire one functions, Real Anal. Exchange 21 (1995-96), 165-174.

[15] Goffman, C., Real Functions, Holt, Reinhart, Winston, New York, 1964.

[16] Gruber, P. M., Dimension and structure of typical compact sets, continua and curves, Monatsh. Math. 108 (1989), 149-164.

[17] Repický, M., An example which discerns porosity and symmetric porosity, Real Anal. Exchange 17 (1991-92), 416-420.

[18] Vallin, R. W., An introduction to shell porosity, Real Anal. Exchange 18 (1992-93), 294-320.

[19] Zajíček, L., Sets of $\sigma$-porosity and sets of $\sigma$-porosity $(q)$, Časopis Pěst. Mat. 101 (1976), 350-359.

[20] Zajíček, L., Porosity and $\sigma$-porosity, Real Anal. Exchange 13 (1987-88), 314-351.

[21] Zajíček, L., On the symmetric and ordinary derivative, Atti Sem. Mat. Fis. Univ. Modena 41 (1993), 263-267. 
Michael J. Evans

Department of Mathematics

WASHINGTON AND LEE UNIVERSITY

LEXINGTON, VIRGINIA 24450

U.S.A.

E-MAIL: MJEVANS@WLU.EDU
Paul D. Humke Department of Mathematics

St. Olaf College

Northfield, Minnesota 45701

U.S.A.

E-MAIL: HUMKE@STOLAF.EDU 